FLÁVIO NUNES SiVINI

Carlos Teixeira Brandt ${ }^{2}$

DJalma Belo Fllho

Artigo original

\title{
Índices de resistência nas artérias ovariana e uterina na fase lútea média em portadoras de esquistossomose mansônica na sua forma hepatoesplênica
}

\author{
Ovarian and uterine arterial resistance indexes on the midluteal \\ phase in patients suffering from schistosomiasis mansoni in its \\ hepatosplenic form
}

\begin{abstract}
Palavras-chave
Artérias/fisiopatologia

Schistosoma mansoni

Ultrassonografia doppler em cores/métodos

Ciclo menstrual

Fase lútea/fisiologia

Fluxo sanguíneo regional

Corpo lúteo

Resistência vascular

Keywords

Arteries/physiopathology

Schistosoma manson

Ultrasonography, doppler, color/methods

Menstrual cycle

Luteal phase/ physiology

Regional blood flow

Corpus luteum

Vascular resistance
\end{abstract}

Correspondência:

Flávio Nunes Sivini

Departamento de Tocoginecologia da Faculdade de Ciências Médicas

da Universidade de Pernambuce

Avenida Boa Viagem, 5.600/302 - Boa Viagem

CEP 51030-000 - Recife (PE), Brasil

Fone: (81) 9972-3567

E-mail: flaviosivinigglobo.com

Recebido

13/5/09

\section{Resumo}

OBJETIVO: avaliar a repercussão da hipertensão porta nos índices de resistência arterial ovariano, uterino e na periferia do corpo lúteo, na fase lútea média do ciclo menstrual. MÉTODOS: em estudo observacional com corte transversal, 28 portadoras de esquistossomose mansônica na forma hepatoesplênica submetidas à esplenectomia e ligadura da veia gástrica esquerda (EHEO), um grupo similar de 28 pacientes não operadas (EHENO) e 29 voluntárias sadias (VS) foram submetidas à doplerfluxometria na fase lútea média do ciclo menstrual. O índice de resistência (RI) de Pourcelot foi usado como referência RI=[(S-D)/S], em que S significa o pico de velocidade sistólica, e D, o fim da diástole. Escolhia-se o melhor traçado no ramo ascendente da artéria uterina, na artéria ovariana, quando acessível, ou na artéria intraovárica. Quando da existência de corpo lúteo, o Rl era medido na periferia do mesmo. Os dados obtidos foram analisados pelos testes de Kruskal-Wallis e Mann-Whitney. RESULTADOS: não houve diferença significativa entre os grupos no que diz respeito às médias dos índices de resistência das artérias ovarianas (Kruskal-Wallis-p=0,50). Foi observada a tendência de que a média dos índices de resistência da artéria uterina direita fosse maior no grupo de EHENO (Kruskal-Wallis-p<0,07); todavia, foi similar no que diz respeito à artéria uterina esquerda (Kruskal-Wallis-p=0, 14). Índices de resistência arterial significativamente mais baixos foram observados na periferia do corpo lúteo, quando comparados com os índices das artérias ovarianas contra laterais em todos os grupos (Mann-Whitney-p<0,000 1). CONCLUSÕES: não se demonstrou diferença entre os grupos no que diz respeito aos índices de resistência das artérias ovarianas e uterinas. A hipertensão porta em portadoras de doença esquistossomótica na forma hepatoesplênica não altera o fenômeno natural de diminuição do índice de resistência arterial no ovário no qual ocorre a ovulação.

\section{Abstract}

PURPOSE: to evaluate the repercussion of portal hypertension in the indexes of the ovarian, uterine and luteus body periphery arterial resistance, at the medium luteal phase of the menstrual cycle. METHODS: in an observational study with transversal cohort, 28 patients with hepatosplenic schistosomiasis mansoni, submitted to splenectomy and ligation of the left gastric vein (HESO), a similar group of 28 patients who had not had the surgery (HESNO) and 29 healthy volunteers $(\mathrm{HV})$ were submitted to Doppler fluxmetry in the medium luteal phase of the menstrual cycle. Pourcelot's resistance index has been used as $R I=[(S-D) / S]$, in which $S$ means the highest systolic speed, and D, the end of diastole. The best record in the ascendant branch of the uterine artery, in the ovarian artery, when accessible, or in the intraovarian artery, was chosen. When the luteus body was present, the RI was measured in its periphery. The data obtained were analyzed by Kruskal-Wallis and Mann-Whitney tests. RESULTS: there was no significant difference among the groups, concerning the mean Rl of the ovarian arteries (Kruskal-Wallis, $\mathrm{p}=0.50$ ). There was a tendency for higher right uterine artery RI in the HESNO group (Kruskal-Wallis, $p<0.07$ ), but it was similar in the left uterine artery (Kruskal-Wallis, $p=0.14$ ). Arterial Rls significantly lower have been observed in the luteus body periphery, when compared to the contralateral ovarian arteries in all the groups (Mann-Whitney, $\mathrm{p}<0.0001$ ). CONCLUSIONS: there was no difference among the groups, regarding the ovarian and uterine Rls. The portal hypertension in patients with hepatosplenic schistosomiasis does not affect the natural phenomenon of arterial RI decrease in the ovary where ovulation occurs.

Hospital das Clínicas do Centro de Ciências da Saúde (CCS) da Universidade Federal de Pernambuco - (UFPE) - Recife (PE), Brasil. 'Professor Adjunto do Departamento de Tocoginecologia da Faculdade de Ciências Médicas da Universidade de Pernambuco - UPE - Recife (PE), Brasil.

2 Professor Titular de Cirurgia Pediátrica do Departamento de Cirurgia do Centro de Ciências da Saúde (CCS) da Universidade Federal de Pernambuco - (UFPE) - Recife (PE), Brasil.

${ }^{3}$ Médico Ultrassonografista do Centro Integrado de Saúde Amaury Medeiros (CISAM) da Universidade de Pernambuco - UPE - Recife (PE), Brasil. 


\section{Introdução}

Desde os primórdios da civilização, a espécie humana convive com a infecção pelo trematódeo Schistosoma mansoni, agente causal da esquistossomose ${ }^{1}$. Apesar de sua atual baixa morbidade no Brasil, essa parasitose continua a constituir importante problema de saúde pública, tendo em vista seu potencial de expansão. Observa-se a ampliação de sua área de transmissão com o surgimento de focos de esquistossomose em áreas antes consideradas indenes. Nos últimos anos, tem sido relatada a urbanização da doença, com surgimento de casos autóctones nas regiões periurbanas das grandes cidades brasileiras ${ }^{1-4}$.

A esquistossomose mansônica tem sido mais conhecida por meio de suas repercussões em vísceras relacionadas direta ou indiretamente ao sistema venoso portal; porém, lesões induzidas pelos ovos no aparelho reprodutivo feminino são importantes complicações da infecção pelo Schistosoma mansoni ${ }^{5}$. As manifestações genitais têm sido negligenciadas como uma entidade patológica. No entanto, estima-se que 6 a 27\% das meninas e mulheres com esquistossomose intestinal, pelo menos temporariamente, sofram de doença induzida pelos ovos sequestrados em algum lugar nos seus órgãos genitais ${ }^{5}$. Nos casos graves, observa-se infertilidade, abortamento, restrição do crescimento intrauterino e prematuridade $^{6-8}$.

Uma das consequências hemodinâmicas da hipertensão porta, devido à fibrose periportal esquistossomótica, consiste no aparecimento do fluxo reverso ou hepatofugal, levando à recanalização de veias e desvios porto-sistêmicos entre as tributárias do sistema porta e a circulação venosa sistêmica. Entre essas comunicações, são destacadas aquelas com a veia renal e a veia cava inferior, fazendo com que ocorra um aumento da resistência à drenagem venosa das veias gonadais para as veias renal esquerda e cava inferior ${ }^{9-11}$. A varicocele ovariana pode acarretar alterações funcionais das trompas e das funções endócrina e exócrina ovarianas ${ }^{11}$.

A congestão e, consequentemente, a estase venosa do plexo pampiniforme, pode provocar alteração da secreção ovariana ${ }^{12}$. Se a reação granulomatosa se desenvolve próxima ao hilo ovariano, a obstrução hilar e aderências paraovarianas podem ser responsáveis pela anovulação ${ }^{13}$.

Ovos, larvas ou depósitos de imunocomplexos antígeno-anticorpo do Schistosoma podem ser encontrados em praticamente qualquer órgão ou tecido do organismo ${ }^{14}$. Localizações incomuns são encontradas frequentemente na forma hepatoesplênica desta parasitose ${ }^{14}$.

A redução do fluxo das artérias uterinas e ovarianas pode estar associada à infertilidade. Índices de resistência significativamente mais baixos foram observados nas artérias uterinas, ovarianas, arcuadas, radiais e espirais na fase média luteal do ciclo menstrual em pacientes que ovulam ${ }^{15-20}$. Em ciclos menstruais ovulatórios normais, os índices de resistência e pulsatilidade arterial ovariano são significantemente mais baixos na fase lútea, quando comparados à fase folicular ${ }^{20,21}$. O estudo da resistência ao fluxo sanguíneo arterial no corpo lúteo auxilia, inclusive, na avaliação de sua adequabilidade funcional ${ }^{22}$.

A inexistência de estudos a respeito de prováveis repercussões hemodinâmicas nos ovários e no útero de portadoras de esquistossomose mansônica na forma hepatoesplênica, na fase lútea do ciclo menstrual, motivou a presente pesquisa.

\section{Métodos}

Foi realizado um estudo observacional, prospectivo e analítico, com corte transversal, no período de Maio de 2005 a Agosto de 2008, no Hospital das Clínicas (HC) do Centro de Ciências da Saúde (CCS) da Universidade Federal de Pernambuco (UFPE).

O protocolo usado nesse estudo foi aprovado pelo Comitê de ética do Centro Integrado de Saúde Amaury de Medeiros (CISAM) da Universidade de Pernambuco (UPE). Os sujeitos do estudo foram informados, em linguagem simples e acessível, de que estariam participando de uma pesquisa clínica, e assinaram o termo de consentimento livre e esclarecido.

As pacientes portadoras de esquistossomose hepatoesplênica (EHE) tinham o diagnóstico firmado através de acompanhamento no ambulatório de Gastroenterologia do Hospital das Clínicas, com exames de fezes, avaliação da função hepática e ultrassonografia abdominal (constatando a presença de fibrose de Symmers). Todas procediam de área endêmica, com histórico familiar sugestivo também com a doença. O Grupo Controle era proveniente da mesma área endêmica e foi submetido a exame clínico e ultrassonog ráfico para afastar o comprometimento hepático característico da EHE.

Oitenta e cinco mulheres foram incluídas e submetidas a um questionário dirigido (anamnese) no qual foram abordados aspectos pertinentes relacionados à doença (esquistossomose) e fatores reprodutivos. Foram divididas em três grupos: 28 pacientes portadoras de esquistossomose, na forma hepatoesplênica, tratadas clinicamente e submetidas à descompressão do sistema porta (esplenectomia e ligadura da veia gástrica esquerda - EHEO); 28 pacientes esquistossomóticas, na forma hepatoesplênica, tratadas clinicamente, mas não operadas (EHENO); 29 mulheres não esquistossomóticas (voluntárias sadias) oriundas da mesma zona endêmica e de condição socioeconômica semelhante (VS).

Todos os grupos demonstraram interesse em investigar a possibilidade da existência de ciclos menstruais 
ovulatórios (mantendo, dessa forma, sua capacidade reprodutora em relação à função hormonal ovariana). Foram excluídas as pacientes que estavam fora do período reprodutivo (menacme); mulheres que haviam feito uso de anticoncepcionais hormonais nos últimos três meses; as que tinham história de esterilidade e irregularidade do ciclo menstrual (intervalos maiores ou menores que 26-30 dias); aquelas com antecedentes de doenças sistêmicas (hipertensão arterial, diabetes mellitus, acidente vascular cerebral, cardiopatias etc.) ou ginecológicas. Foi excluída uma paciente no grupo das operadas cuja ultrassonografia constatou a presença de mioma.

A doplervelocimetria foi realizada pelo mesmo examinador, na fase lútea média do ciclo menstrual. Foram determinados os índices de resistência das artérias uterinas, ovarianas e na periferia do corpo lúteo (quando presente). Como referência, foi usado o índice de resistência de Pourcelot $\mathrm{RI}=[(\mathrm{S}-\mathrm{D}) / \mathrm{S}]$. O tipo de endométrio também foi avaliado pelo método (secretor ou proliferativo). Foi utilizado o aparelho GE, modelo Voluson 730, com transdutor de banda larga multifrequencial com variação de 5 a $9 \mathrm{MHz}$.

A ultrassonografia transvaginal foi realizada com a bexiga vazia, para que os órgãos pélvicos ficassem na zona focal do transdutor e para se evitar o efeito da distensão vesical sobre as artérias uterinas. O exame foi realizado com a paciente em posição supina, joelhos fletidos e quadris ligeiramente elevados.

Foi colocado um preservativo com gel no transdutor antes de inseri-lo na vagina, protegendo, desse modo, a paciente e o transdutor. Foram obtidos cortes longitudinais, oblíquos e transversais dos órgãos pélvicos. Escolhia-se o melhor traçado em nível do ramo ascendente da artéria uterina e, em relação aos ovários, a artéria ovariana, quando acessível, ou a artéria intraovárica. Quando da existência de corpo lúteo, o IR arterial era medido na periferia do mesmo. Dependendo da direção do fluxo em relação ao transdutor, as cores do Doppler poderiam ser arbitrariamente escolhidas. Neste estudo, foi convencionado que o fluxo em direção ao transdutor teria cor vermelha (arterial) e, ao se afastar, a cor seria azul (venoso). Nas artérias, havia modificação das velocidades sanguíneas durante o ciclo cardíaco, com velocidades maiores na sístole do que na diástole; nas veias, o fluxo era contínuo.

Para análise estatística, foi utilizado o software Graphpad instat 3.06. Os dados quantitativos foram resumidos por suas médias e desvios padrão. Foi usado o teste de Kruskal-Wallis para avaliação de possíveis diferenças entre médias de variáveis contínuas nos três grupos estudados, e o teste Mann-Whitney na avaliação entre dois grupos. Foi adotado o intervalo de confiança de $95 \%$, e valores de $\mathrm{p} \leq 0,05$ foram considerados significantes.

\section{Resultados}

Os índices de resistência em conjunto, nas artérias ovariana direita (AOD) e esquerda (AOE), variaram respectivamente entre: $0,3-1,0$ no grupo de pacientes não operadas; $0,3-1,0$ no grupo de pacientes operadas; e 0,4-1,0 no Grupo Controle. Não houve diferença significativa entre os grupos em relação às médias dos índices de resistência das artérias ovariana direita e esquerda (Kruskal-Wallis-p=0,5) (Tabela 1).

Os índices de resistência nas artérias uterinas direita (AUD) e esquerda (AUE) variaram, respectivamente, entre: 0,4-1,0, e 0,7-1,0 no grupo de pacientes não operadas; 0,61,0 , e $0,5-1,0$ no grupo de pacientes operadas; e 0,7-1,0, e 0,7-1,0 no Grupo Controle. Não foi observada diferença significativa entre as médias dos índices de resistência das artérias uterinas direita e esquerda (Kruskal-Wallis - $\mathrm{p}<0,07$ e $\mathrm{p}=0,14)$. Apenas uma tendência foi verificada em relação aos índices entre o grupo de EHEO e EHENO nas artérias uterinas ao lado direito (Tabela 2).

Os índices de resistência nas artérias ovarianas na periferia do corpo lúteo e nos ovários contralaterais variaram, respectivamente, entre: $0,3-0,6$, e $0,5-1,0$ no grupo de pacientes não operadas; $0,3-0,4$, e $0,5-1,0$ no grupo de pacientes operadas; e 0,4-0,4, e 0,4-1,0 no Grupo Controle. As médias dos índices de resistência arterial foram significantemente mais baixas nas artérias na periferia do corpo lúteo do que nos ovários contralaterais em todos os grupos (Mann-Whitney-p<0,0001) (Tabela 3).

Tabela 1 - Médias e medianas dos índices de resistência das artérias ovariana direita e esquerda

\begin{tabular}{|c|c|c|c|c|c|c|}
\hline Grupo & n & $\bar{\chi} \pm \mathbf{D P}$ & Mediana & Mínimo & Máximo & Valor de $p$ \\
\hline EHEO & 28 & $0,56 \pm 0,17$ & 0,52 & 0,35 & 1,0 & \\
\hline EHENO & 28 & $0,52 \pm 0,10$ & 0,51 & 0,35 & 1,0 & \\
\hline VS & 29 & $0,57 \pm 0,17$ & 0,52 & 0,41 & 1,0 & \\
\hline
\end{tabular}

$\bar{\chi}$ : média; DP: desvio padrão; $\mathrm{AO}$ : artérias ovarianas. 
Tabela 2 - Médias e medianas dos índices de resistência das artérias uterina direita e esquerda

\begin{tabular}{|c|c|c|c|c|c|c|}
\hline Grupo & n & $\bar{\chi} \pm \mathrm{DP}$ & Mediana & Mínimo & Máximo & Valor de $\mathrm{p}$ \\
\hline AUD & & & & & & 0,07 \\
\hline EHEO & 28 & $0,78 \pm 0,07$ & 0,76 & 0,72 & 1,0 & \\
\hline vS & 29 & $0,80 \pm 0,09$ & 0,79 & 0,70 & 1,0 & \\
\hline Total & 85 & & & & & \\
\hline EHENO & 28 & $0,83 \pm 0,06$ & 0,83 & 0,75 & 1,0 & \\
\hline vs & 29 & $0,82 \pm 0,08$ & 0,80 & 0,71 & 1,0 & \\
\hline Total & 85 & & & & & \\
\hline
\end{tabular}

$\bar{\chi}$ : média; DP: desvio padrão; AUD: artéria uterina direita; QUE: artéria uterina esquerda.

Tabela 3 - Médias e medianas dos índices de resistência arterial na periferia do corpo lúteo e nos ovários contralaterais

\begin{tabular}{lccccc}
\hline Grupo & n & $\begin{array}{c}\text { Ovário com Clúteo } \\
\bar{x} \pm \text { DP }\end{array}$ & Mediana & $\begin{array}{c}\text { Ovário contralateral } \\
\bar{\chi} \pm \text { DP }\end{array}$ & Mediana \\
\hline EHE0 & 22 & $0,43 \pm 0,03$ & $0,43(0,35-0,48)$ & $0,60 \pm 0,14$ & $0,55(0,51-1,0)$ \\
EHENO & 23 & $0,44 \pm 0,05$ & $0,45(0,35-0,49)$ & $0,57 \pm 0,11$ & $0,53(0,50-1,0)$ \\
VS & 20 & $0,43 \pm 0,02$ & $0,42(0,40-0,47)$ & $0,67 \pm 0,18$ & $0,58(0,44-1,0)$ \\
Total & 65 & & & & 0,000 \\
\hline
\end{tabular}

$\bar{\chi}$ : média; DP: desvio padrão; C lúteo: corpo lúteo.

\section{Discussão}

Nos ovários de mulheres sadias, com ciclos menstruais normais, os índices de resistência arterial são significativamente mais baixos no ovário em que ocorre a ovulação, fenômeno compatível com o aumento do fluxo sanguíneo ${ }^{17,18,23-27}$.

Observação semelhante ocorreu no estudo atual, tanto em relação às portadoras de esquistossomose mansônica na forma hepatoesplênica, operadas ou não, quanto entre mulheres que constituíram o Grupo Controle (sem a doença). De fato, ao se comparar as médias dos índices de resistência arterial nos ovários onde ocorreu a ovulação (periferia do corpo lúteo) com as médias dos índices de resistência nas artérias dos ovários contralaterais, foi observada, em concordância com outros autores ${ }^{17,18,23-27}$, diferença significativa em todos os grupos.

A diminuição nos índices de resistência arterial é determinada pela neovascularização formada ao redor do folículo dominante e, subsequentemente do corpo lúteo, e pela vasodilatação induzida pelo estradiol nas artérias ovarianas $^{28}$. A hipertensão porta em portadoras de esquistossomose hepatoesplênica não parece impedir que os novos capilares desenvolvidos ao redor do folículo em crescimento, e no corpo lúteo formado posteriormente, diminuam a impedância ao fluxo nos ovários na fase folicular tardia e lútea media ${ }^{23,28}$. Por outro lado, estudando os índices arteriais ovarianos como um todo, não foi observada diferença estatística entre os grupos.
Vários estudos permitiram conhecer as modificações na vascularização útero-ovariana com o uso da doplervelocimetria ${ }^{17-19,23}$. O fluxo sanguíneo aumenta significativamente nas artérias uterinas e seus ramos na fase lútea ${ }^{17}$. De fato, índices de resistência mais baixos são vistos nas artérias uterinas de mulheres que ovulam na fase lútea média ${ }^{15,16,24}$. Esse declínio na resistência arterial uterina durante a fase lútea reflete adequada vascularização para a implantação do blastocisto ${ }^{15,16,24}$.

Existe referência à diminuição nos índices de resistência na artéria uterina ipsilateral ao lado onde ocorreu à ovulação ${ }^{25} \mathrm{ou}$, simplesmente, envolvimento pequeno (marginal) nas mudanças de impedância durante a fase lútea do ciclo menstrual ${ }^{26}$. Entretanto, essa variação nos índices de resistência das artérias uterinas durante o ciclo menstrual normal é assunto contraditório, considerando que a diferença entre esses índices, em relação ao lado do folículo ovariano dominante, não é observada com unanimidade ${ }^{24}$.

No presente estudo, em todos os grupos foram observados índices de resistência nas artérias uterinas ipsilaterais menores do que os encontrados nas artérias uterinas contralaterais, porém, sem significância. Foi observado que, no grupo de operadas, pacientes, portanto, submetidas à cirurgia descompressiva (esplenectomia e ligadura da veia cava esquerda), as médias foram mais baixas nas artérias ipsilaterais $(0,77 \pm 0,08$ versus $0,82 \pm 0,07$ no grupo de não operadas), como se o fenômeno da descompressão venosa pudesse, de alguma maneira, influenciar um fenômeno 
puramente arterial. Por outro lado, também não houve significância nesta diferença.

Nas pacientes EHENO foi observada a tendência de que a média dos índices de resistência fosse maior nas artérias uterinas do lado direito quando comparada ao grupo de operadas, porém, sem nenhuma relação com o lado do ovário em que ocorreu a ovulação. As pacientes não operadas, ou seja, não submetidas à cirurgia de descompressão, estariam mais sujeitas a uma maior resistência ao fluxo sanguíneo, mesmo se tratando de sangue arterial. Por outro lado, o estudo da resistência vascular nas artérias uterinas não avalia, necessariamente, apenas os fenômenos envolvidos no território uterino. Devido ao arco arterial formado pela anastomose com a artéria ovariana, fenômenos ovarianos, como desenvolvimento folicular e síntese de esteróides, também podem influenciar o comportamento do fluxo nesses vasos.

Este estudo chama a atenção para a importância da avaliação dos índices de resistência arterial ovarianos na fase lútea do ciclo menstrual. Índices baixos, traduzindo fluxo sanguíneo adequado, permitem prever ciclo ovulatório com possibilidade de uma futura gravidez, se desejada. A esquistossomose mansônica, na forma hepatoesplênica, em pacientes operadas ou não, não alterou os índices de resistência arterial ovariano e uterino na fase lútea do ciclo menstrual, quando estes foram comparados aos do Grupo Controle.

\section{Referências}

1. Gargioni C, Silva RM, Thomé CM, Quadros CMS, Kanamura HY. Utilização de método sorológico como ferramenta diagnóstica para implementação da vigilância e controle da esquistossomose no Município de Holambra, São Paulo, Brasil. Cad Saúde Pública. 2008;24(2):373-9.

2. Katz N, Peixoto SV. Análise crítica da estimativa do número de portadores de esquistossomose mansoni no Brasil. Rev Soc Bras Med Trop. 2000;33(3):303-8.

3. Resendes APC, Souza-Santos R, Barbosa CS. Internação hospitalar e mortalidade por esquistossomose mansônica no Estado de Pernambuco, Brasil, 1992/2000. Cad Saúde Pública. 2005;21(5):1392-401.

4. Graeff-Teixeira C, Anjos CB, de Oliveira VC, Velloso CF, Fonseca MB, Valar $C$, et al. Identification of a transmission focus of Schistosoma mansoni in the southernmost Brazilian State, Rio Grande do Sul. Mem Inst Oswaldo Cruz. 1999;94(1):9-10.

5. Feldmeier H, Daccal RC, Martins M, Soares V, Martins R. Genital manifestations of schistosomiasis mansoni in women: important but neglected. Mem Inst Oswaldo Cruz. 1998;93 Suppl 1:127-33.

6. Bahrami S, Alatassi H, Slone SP, O'Connor DM. Tubal gestation and schistosomiasis: a case report. J Reprod Med. 2006;5 1 (7):595-8.

7. Bourée P, Bisaro F. Parasitic diseases and pregnancy. Rev Prat. 2007;57(2): 137-47.

8. Swai B, Poggensee G, Mtweve S, Krantz I. Female genital schistosomiasis as an evidence of a neglected cause for reproductive ill-health: a retrospective histopathological study from Tanzania. BMC Infect Dis. 2006;6:134.

9. Kennedy A, Hemingway A. Radiology of ovarian varices. $\mathrm{Br} \mathrm{J}$ Hosp Med. 1990;44(1):38-43.

10. Chagas HM, Brandt CT, Jucá M, Wyszomirska RMAF, Costa BLN, Montoro MVS. Varizes de ovário em portador de esquistossomose hepato-esplênica. An Fac Med Univ Fed Pernamb. 2002;47(2):91-6.

11. Giacchetto C, Catizone F, Cotroneo GB, Cavallaro V, Cammisuli $F$, Minutolo $V$, et al. Radiologic anatomy of the genital venous system in female patients with varicocele. Surg Gynecol Obstet. 1989; 169(5):403-7.

12. Spanio P. Importanza del varicocele pelvico nella patologia dell'apparato genitale femminile. Minerva Ginecol. 1975;27(3):262-7.

13. Helling-Giese G, Sjaastad A, Poggensee G, Kjetland EF, Richter J, Chitsulo L, et al. Female genital schistosomiasis (FGS): relationship between gynecological and histopathological findings. Acta Trop. 1996;62(4):257-67.

14. Oréfice F, Pittela JEH, Simal CJR, Coscarelli G. Uveite esquistossomótica: alterações fundoscópicas; achados histológicos do ovo do S. mansoni, abordagem da etiologia e tratamento. Arq Bras Oftalmol. 1988;51(3): 123-34.

15. Goswamy RK, William G, Steptoe PC. Decreased uterine perfusion-a cause of infertility. Hum Reprod. 1988;3(8):955-9.

16. Goswamy RK, Steptoe PC. Doppler ultrasound studies of the uterine artery in spontaneous ovarian cycles. Hum Reprod. 1988;3(6):721-6.

17. Salle B, Gaucherand P, Rudigoz RC. Le Doppler pulsé couleur par voie transvaginal dans l'étude du cycle menstrual. J Gynecol Obstet Biol Reprod (Paris). 1994;23(7):767-71.

18. Thaler I, Manor D, Rottem S, Timor-Tritsch IE, Brandes JM, Itskovitz J. Hemodynamic evaluation of the female pelvic vessels using a high-frequency transvaginal image-directed Doppler system. J Clin Ultrasound. 1990; 18(4):364-9.

19. Kurjak A, Zalud I. Transvaginal color Doppler for evaluating gynecologic pathology of the pelvis. Ultraschall Med. 1990;1 1(4):164-8.

20. Miyazaki T, Tanaka M, Miyakoshi K, Minegishi K, Kasai K, Yoshimura Y. Power and colour Doppler ultrasonography for the evaluation of the vasculature of the human corpus luteum. Hum Reprod. 1998;13(10):2836-41.

21. Mercé LT, Bau S, Bajo JM. Doppler study of arterial and venous intraovarian blood flow in stimulated cycles. Ultrasound Obstet Gynecol. 2001;18(5):505-10.

22. Tamura H, Takasaki A, Taniguchi K, Matsuoka A, Shimamura K, Sugino N. Changes in blood-flow impedance of the human corpus 
luteum throughout the luteal phase and during early pregnancy. Fertil Steril. 2008;90(6):2334-9.

23. Kurjak A, Kupesic-Urek S, Schulman H, Zalud I. Transvaginal color flow Doppler in the assessement of ovarian and uterine blood flow in infertile women. Fertil Steril. 1991;56(5):870-3.

24. Dal J, Vural B,Caliskan E, Ozkan S, Yucesoy I. Power Doppler ultrasound studies of ovarian, uterine, and endometrial blood flow in regularly menstruating women with respect to luteal phase defects. Fertil Steril. 2005;84(1):224-7.

25. Sladkevicius P, Valentin L, Marsál K. Blood flow velocity in the uterine and ovarian arteries during the normal menstrual cycle. Ultrasound Obstet Gynecol. 1993;3(3): 199-208.
26. Scholtes MC, Wladimiroff JW, van Rijen HJ, Hop WC. Uterine and ovarian flow velocity waveforms in the normal menstrual cycle: a transvaginal Doppler study. Fertil Steril. 1989;52(6):981-5.

27. Hata K, Hata T, Senoh D, Makihara K, Aoki S, Takamiya O, et al. Change in ovarian arterial compliance during the human menstrual cycle assessed by Doppler ultrasound. Br J Obstet Gynaecol. 1990;97(2):163-6.

28. Schurz B, Schön HJ, Wenzl R, Eppel W, Huber J, Reinold E. Endovaginal Doppler flow measurements of the ovarian artery in patients with a normal menstrual cycle and with polycystic ovary during in vitro fertilization. J Clin Ultrasound. 1993;21(1): 19-24. 